\title{
Risk factors and medical costs for healthcare-associated carbapenem-resistant Escherichia coli infection among hospitalized patients in a Chinese teaching hospital
}

Xiujuan Meng, Sidi Liu, Juping Duan, Xun Huang, Pengcheng Zhou, Xinrui Xiong, Ruie Gong, Ying Zhang, Yao Liu, Chenchao Fu, Chunhui Li and Anhua Wü

\begin{abstract}
Background: The emergence and spread of Carbapenem-resistant Escherichia coli (CREC) is becoming a serious problem in Chinese hospitals, however, the data on this is scarce. Therefore, we investigate the risk factors for healthcare-associated CREC infection and study the incidence, antibiotic resistance and medical costs of CREC infections in our hospital.

Methods: We conducted a retrospective, matched case-control-control, parallel study in a tertiary teaching hospital. Patients admitted between January 2012 and December 2015 were included in this study. For patients with healthcare-associated CREC infection, two matched subject groups were created; one group with healthcareassociated CSEC infection and the other group without infection.
\end{abstract}

Results: Multivariate conditional logistic regression analysis demonstrated that prior hospital stay ( $<6$ months) (OR: 3.96; 95\%Cl:1.26-12.42), tracheostomy (OR:2.24; 95\%Cl: 1.14-4.38), central venous catheter insertion (OR: 8.15; 95\%Cl: 2.31-28.72), carbapenem exposure (OR: 12.02; 95\%Cl: 1.52-95.4), urinary system disease (OR: 16.69; 95\%Cl: 3.01-89. 76), low hemoglobin (OR: 2.83; 95\%Cl: 1.46-5.50), and high blood glucose are associated (OR: 7.01; 95\%Cl: 1.89-26. 02) with CREC infection. Total costs $(p=0.00)$, medical examination costs $(p=0.00)$, medical test costs $(p=0.00)$, total drug costs $(p=0.00)$ and ant-infective drug costs $(p=0.00)$ for the CREC group were significantly higher than those for the no infection group. Medical examination costs $(p=0.03)$, total drug costs $(p=0.03)$, and anti-infective drug costs $(p=0.01)$ for the CREC group were significantly higher than for the CSEC group. Mortality in CREC group was significantly higher than the CSEC group $(p=0.01)$ and no infection group ( $p=0.01)$.

Conclusion: Many factors were discovered for acquisition of healthcare-associated CREC infection. CREC isolates were resistant to most antibiotics, and had some association with high financial burden and increased mortality.

Keywords: Healthcare-associated infection, Risk factors, CREC, CSEC

\footnotetext{
*Correspondence: lichunhui@csu.edu.cn; xywuanhua@csu.edu.cn Infection Control Centre, Xiangya Hospital of Central South University, Changsha, China
} 


\section{Background}

Carbapenems have long served as reliable and potent agents against Gram-negative bacilli [1]. Carbapenems are most consistently active against members of the Enterobacteriaceae family [2], however, few treatment options exist for carbapenem-resistant Enterobacteriaceae (CRE) infection, which can result in high mortality [3]. In recent years, carbapenem-resistant Escherichia coli (CREC), as one class of CRE, has become a major threat in hospitals worldwide [4-7]. Carbapenem resistance in E.coli is an emerging problem that is mainly caused by plasmid-encoded carbapenemases [8-13]. As a result of the emergence of carbapenemases [14], antimicrobial resistance is increasing in most hospitals, and has become a global healthcare problem. CREC strains should be closely monitored because of their potential trend to spread in both hospital and community settings [15].

There are several previous studies on the risk factors for CRE infection [16, 17], but few published studies have specifically evaluated the risk factors for CREC acquisition, especially in China. Therefore, we performed a retrospective study to evaluate the risk factors for healthcare-associated infection (HAI) caused by CREC among in-patients in a teaching hospital in central south China, thus, we could do better in decreasing the incidence of CREC infection.

The case-control-control study design of this study, which utilizes two separate case-control analyses, has become a standard method for the specific identification of risk factors that are uniquely connected to infection by antimicrobial-resistant pathogens $[18,19]$. We studied the risk factors for CREC infection through the case-control-control design. In addition, CREC is often resistant to multiple antibiotics; therefore, we investigated the antibiotic resistance and economic burden of CREC infections.

\section{Methods}

\section{Study design and setting}

We conducted a retrospective, parallel, case-control-control study to identify the incidence, risk factors, antibiotic resistance, and medical costs associated with the acquisition of healthcare-associated CREC infection among hospitalized patients treated at Xiangya Hospital, a 3500bed general hospital in Changsha, Hunan Province, Central South China. The CREC infection group was compared with a no infection group to assess the risk factors for acquisition of CREC infection; meanwhile, the CREC group was compared with the CSEC infection group to evaluate reasons for antibiotic resistance.

Subjects with CREC or CSEC isolated from multiple sites, or on multiple dates, were counted only once, and the data from the first infection was included in the study. Healthcare-acquired CREC or CSEC infection was defined as isolation 48 hours after admission to the hospital. Healthcare-associated infection (HAI) was defined according to the CDC/NHSN surveillance criteria in patients with samples from any specimen source site positive for CR-EC or CS-EC; meanwhile, the patients with CR-EC or CS-EC colonization and communityassociated infection (CAI) were ruled out.

\section{Study population}

Patients from whom CREC were isolated from clinical cultures from any source between January 1, 2012 and December 31, 2015 were included in this study. For each CREC patient, we randomly selected two controls from hospitalized patients who were admitted within the same period with CSEC isolated, and the two groups were matched for age and sex. Additionally, we selected two controls from the in-patients admitted within the study period with no bacterial infection, and the two groups were matched for age and sex.

\section{Microbiological identification and susceptibility testing}

An automated broth microdilution method (Vitek 2; bioMérieux, Marcy-l'Étoile, France) was used to perform identification and susceptibility testing. Carbapenem resistance was determined using the disk diffusion method. All isolates with resistance, or intermediate susceptibility to carbapenem were defined as resistant isolates. Clinical and Laboratory Standards Institute document M100-S22 (January 2012) was used for interpretation of the antimicrobial susceptibility testing and ESBL testing, and CREC was defined as E.coli resistant to at least one of the carbapenems (imipenem, meropenem, or ertapenem).

Using current EUCAST breakpoints, imipenem MICs of CR-KP isolates ranged from 2 to $>32 \mu \mathrm{g} / \mathrm{ml}$ (breakpoint for resistance and intermediate susceptibility $\mathrm{MIC} \geq 2 \mu \mathrm{g} / \mathrm{ml}$ ); meropenem MICs from 4 to $>32 \mu \mathrm{g} / \mathrm{ml}$ (breakpoint for resistance and intermediate susceptibility $\mathrm{MIC} \geq 4 \mu \mathrm{g} / \mathrm{ml}$ ); all the isolates had ertapenem MICs in the resistant range (breakpoint for resistance and intermediate susceptibility $\mathrm{MIC} \geq 1 \mu \mathrm{g} / \mathrm{ml}$ ).

\section{Data collection}

Data were obtained from patients' medical records, and relative data were recorded on structured abstraction forms. Variables analyzed as possible predictors included demographics (age, sex, marital status, and ward class); clinical departments where strains were isolated; and the history of admission before the infection (within 6 months prior to E.coli infection); length of hospital, intensive care unit (ICU) stay before E.coli infection; specimen source site (blood, bile, etc.); invasive procedures (urinary catheter insertion, mechanical ventilation, etc.) within 1 month 
prior to E.coli infection; surgical procedures within 1 month prior to E.coli infection; administration of drugs (glucocorticoids and immunosuppressive agents), radiotherapy and chemotherapy within 1 month prior to E.coli infection; specific co-morbidities included many system diseases (respiratory, central nervous, etc.); exposure (greater than, or equal to, one day) to antimicrobials (cephalosporins, carbapenems, etc.) within 3 months prior to CREC identification.

We also noted any related laboratory results when healthcare-aquired isolation of E.coli was recorded in the inspection system, and recorded the drug sensitivity test results obtained from the microbiology laboratory and the economic costs associated with these patients as noted in the financial system. The economic costs included total costs, medical examination costs, medical test costs, total drug costs and anti-infective drug costs.

\section{Statistical analysis}

Continuous variables were presented as mean $\pm \mathrm{SD}$, and we used t-tests for comparisons. As the results of the age and average costs of the data for the three groups showed non-normal distribution, they were compared with the median, and the data for two groups were compared using the Wilcoxon rank-sum test. We presented categorical variables as numbers and percentages, and compared percentages using the chi-square test or Fisher's exact test.

We performed univariate analyses for each of the variables using conditional logistic regression to compare the cases and controls in terms of risk factor analysis. The association between independent variables is shown as the odds ratio with $95 \%$ confidence intervals, and variables for which the $P$ value was less than 0.05 in the univariate analysis were included in a conditional logistic regression model for multivariate analysis. Multivariate logistic regression models were used to compare each case group and control group. A forward elimination process was used, and adjusted odds ratios and 95\% confidence intervals were calculated.

A two-tailed $P$ value of less than 0.05 was considered to show statistical significance, and statistical analyses were performed using SPSS 17.0 (SPSS, Inc, Chicago, IL, USA).

\section{Results}

\section{Incidence of CREC infection}

During the 4-year study period, CREC was isolated from 49 patients who met the criteria for healthcareassociated infection (HAI), including fourteen patients in $2012(0.13 / 10,000$ patient days), seventeen patients in $2013(0.15 / 10,000$ patient days), eight patients in 2014 $(0.06 / 10,000$ patient days), and ten patients in 2015 (0.10/10,000 patient days). The incidence of CREC infection over the 4-year study presented in Fig. 1.

\section{Specimen source site and specimen source ward}

A total of 49 patients were included in the case group. CREC was most frequently recovered from respiratory secretions $(28.6 \%)$, followed by urine $(24.5 \%)$, surgical wounds (20.4\%), blood (12.2\%), ascitic fluid (12.2\%), and bile (2.0\%) (Fig. 2). When a positive culture result was obtained, patients infected with CREC were most frequently staying in surgical wards $(46.9 \%)$, followed by medical wards (20.4\%), pediatric wards (16.3\%), ICU wards (12.2\%), and the transplant center (4\%) (Fig. 3).

\section{Resistance rate to antibiotics}

The antibiotic susceptibility patterns of the isolates from the case and control patients are shown in Table 1. All

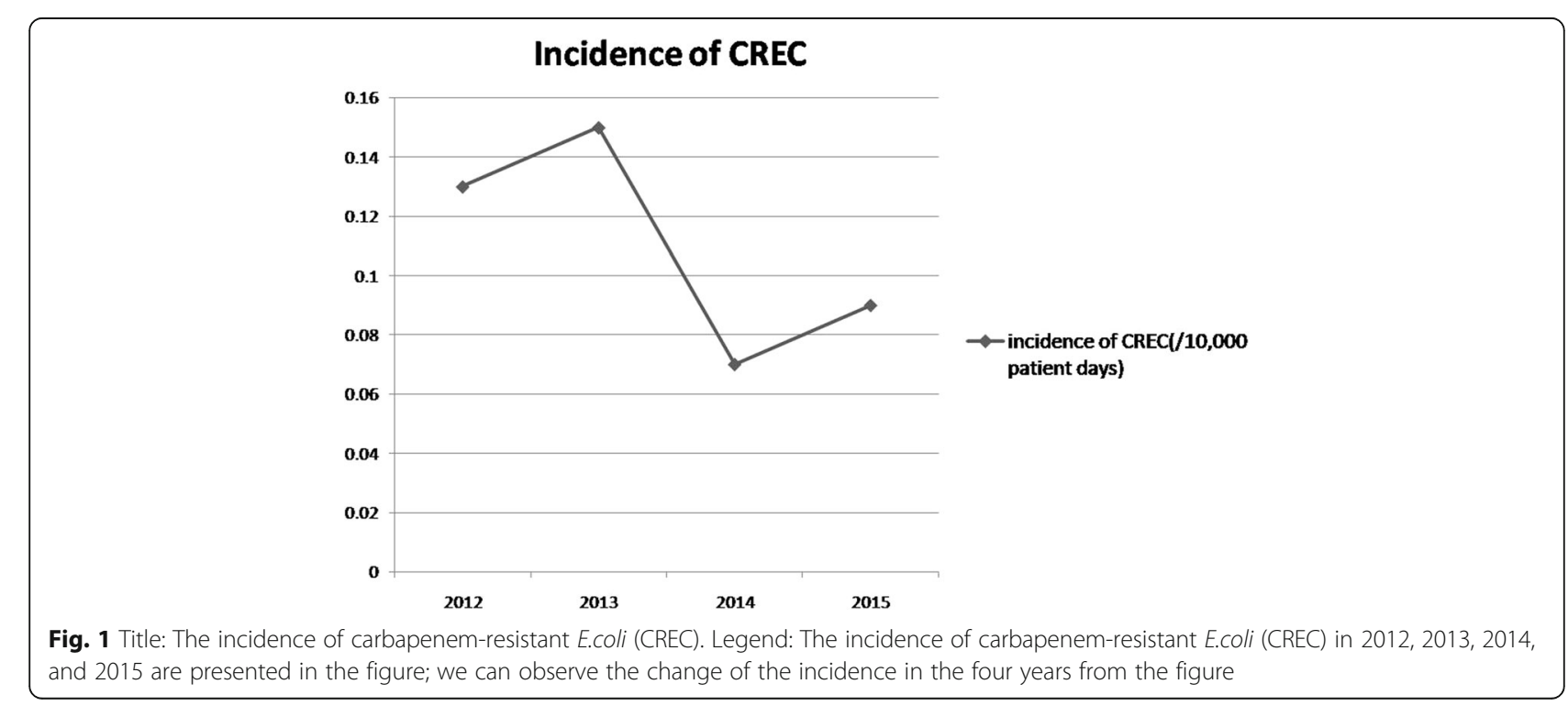




\section{Infection sites}

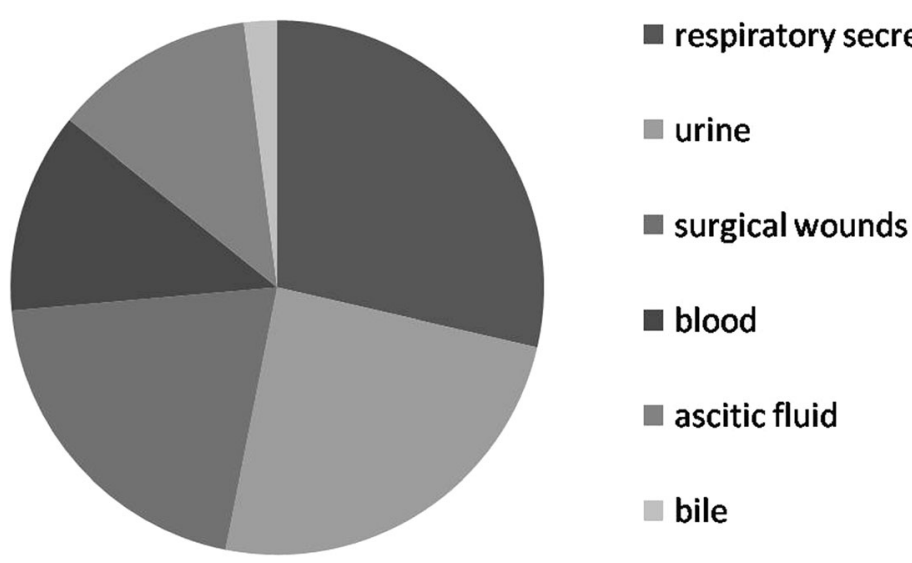

Fig. 2 Title: The infection sites of carbapenem-resistant E.coli (CREC). Legend: The proportion of carbapenem-resistant E.coli (CREC) strains recovered from the sites are presented in the figure, we can observe the regularity of the pathogens distributed

CREC strains were resistant to ampicillin, ampicillin- sulbactam, cefazolin, ceftriaxone, and cefepime, followed by aztreonam, ceftazidime, ciprofloxacin, levofloxacin, piperacillin/tazobactam, trimethoprim and sulfamethoxazole, cefotetan, cefoperazone/sulbactam, tobramycin, and gentamicin; drug resistance rate to nitrofurantoin and amikacin was relatively low.

\section{Univariate and multivariateanalyses regarding the risk factors of the CREC and CSEC groups}

Results of the univariate and multivariate analyses from the comparison of the CREC and CSEC groups regarding the risk factors for healthcare-acquired CREC are shown in Table 2. The univariate conditional logistic regression analysis demonstrated that prior hospital stay ( $<6$ months), urinary catheter insertion, tracheostomy, central venous catheter

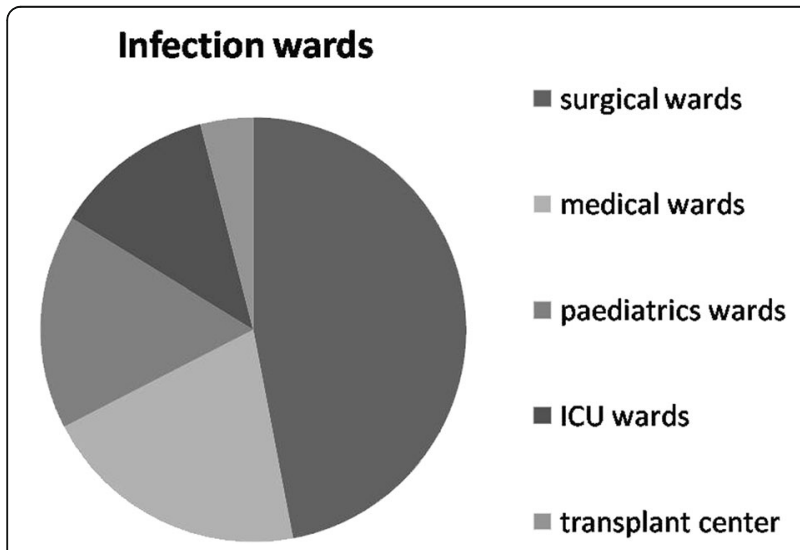

Fig. 3 Title: The infection wards of carbapenem-resistant E.coli (CREC). Legend: The proportion of carbapenem-resistant E.coli (CREC) strains collected in which ward are presented in this figure, we can observe the regularity of the pathogens distributed insertion, gastric tube insertion, urinary system disease, cephalosporins exposure, carbapenems exposure, antifungal agents exposure, glycopeptides and oxazolidinones exposure, low hemoglobin, low blood albumin, and high blood glucose were all risk factors for healthcare-acquired CREC infection. The multivariate conditional logistic regression analysis demonstrated that prior hospital stay ( $<6$ months), incision of trachea, central venous catheter insertion, urinary system disease, low hemoglobin, and high blood glucose were all risk factors for healthcare-acquired CREC infection.

\section{Univariate and multivariate analyses regarding the risk factors in the CREC and no infection groups}

The univariate and multivariate analyses results of the CREC and no infection groups are presented in Table 3. The univariate analysis results showed that prior hospital stay, ICU stay, operation history, urinary catheter insertion, mechanical ventilation, tracheostomy, central venous catheter insertion, bronchofiberscope use, gastric tube insertion, wound drainage tube use, urinary system disease, surgical trauma, cephalosporins exposure, carbapenem exposure, antifungal agents exposure, glycopeptides and oxazolidinones exposure, high white blood cell count, low hemoglobin, low blood albumin, and high blood glucose were all risk factors for CREC infection. Multivariate conditional logistic regression analysis demonstrated that, urinary catheter insertion, central venous catheter insertion and carbapenem exposure were all risk factors associated with the acquisition of CREC.

\section{Medical costs and mortality of the three groups}

Comparison of the CREC and CSEC groups, and the CREC and no infection groups, in terms of economic costs are shown in Table 4. Mortality in the CREC group 
Table 1 The antibiotic-resistanceof the two groups \{carbapenem-resistant E.coli (CREC) and carbapenem-susceptible E.coli (CSEC)\}

\begin{tabular}{llll}
\hline & Case $(n=49)$ & Control $(n=98)$ & $p$ \\
\hline Ampicillin & $47 / 47(100 \%)$ & $90 / 96(94 \%)$ & 0.08 \\
Piperacillin/tazobactam & $38 / 48(79 \%)$ & $10 / 96(10 \%)$ & 0.00 \\
Ampicillin/sulbactam & $40 / 40(100 \%)$ & $75 / 88(85 \%)$ & 0.01 \\
Cefoperazone/sulbactam & $29 / 45(64 \%)$ & $13 / 96(14 \%)$ & 0.00 \\
Cefazolin & $42 / 42(100 \%)$ & $75 / 92(82 \%)$ & 0.00 \\
Ceftazidime & $37 / 39(95 \%)$ & $48 / 87(55 \%)$ & 0.00 \\
Ceftriaxone & $47 / 47(100 \%)$ & $75 / 97(77 \%)$ & 0.00 \\
Cefepime & $49 / 49(100 \%)$ & $50 / 96(52 \%)$ & 0.00 \\
Cefotetan & $22 / 31(71 \%)$ & $2 / 85(2 \%)$ & 0.00 \\
Aztreonam & $47 / 49(96 \%)$ & $62 / 97(54 \%)$ & 0.00 \\
Tobramycin & $26 / 45(58 \%)$ & $51 / 94(54 \%)$ & 0.69 \\
Amikacin & $3 / 48(6 \%)$ & $8 / 98(8 \%)$ & 0.68 \\
Gentamicin & $26 / 48(54 \%)$ & $49 / 98(50 \%)$ & 0.64 \\
Ciprofloxacin & $41 / 46(89 \%)$ & $64 / 96(67 \%)$ & 0.00 \\
Levofloxacin & $41 / 49(84 \%)$ & $60 / 96(63 \%)$ & 0.01 \\
Trimethoprim + sulfamethoxaz ole & $36 / 49(73 \%)$ & $58 / 96(60 \%)$ & 0.05 \\
Nitrofurantoin & $18 / 44(41 \%)$ & $20 / 98(20 \%)$ & 0.01 \\
\hline NOTE Categon &
\end{tabular}

NOTE. Categorical variables are no/total no (\%), case is carbapenem-resistant E.coli (CREC), control is carbapenem-susceptible E.coli (CSEC)

was significantly higher than that in the other two groups. In addition, medical costs of CREC group (including total costs, medical examination costs, medical test costs and total drug costs and anti-infective drug costs) were statistically significantly higher than those for the no infection group. The medical examination costs, and total drug costs and anti-infective drug costs for the CREC group were also statistically significantly higher than those for the CSEC group.

\section{Discussion}

To our knowledge, few studies have evaluated the risk factors for the acquisition of CREC infection. Therefore, the aim of our matched case-control-control study was to assess the potential risk factors [20] for the acquisition of CREC in clinical specimens from hospitalized patients and to investigate the incidence, medical costs, and antibiotic resistance of the strains from these infections.

During our study period, the incidence of CREC infection was lower than $1 / 10,000$ patient days; it was likely related to the presence of active antimicrobial stewardship teams in the hospital. Although the incidence of CREC is low in CRE, carbapenem resistance in Escherichia coli is also emerging worldwide; the reasons for the spread of CREC are likely limited infection control and antimicrobial control measures [21].

The CREC strains were resistant to at least three kind of antibiotics, the antibiotic resistance of the CREC group was more severe than that of the CSEC group. Compared with the strains from the CSEC patients, most of those from the CREC patients were resistant to cephalosporins, penicillin, aztreonam, ciprofloxacin, and levofloxacin, but the strains remained relatively susceptible to amikacin and nitrofurantoin. We could not have chosen a better way to treat CREC infections considering the above results and according to individual clinical conditions.

The results of our study show that the CREC group was associated with more expenses than the other two groups, particularly in terms of the medical examination costs, total drug costs, and anti-infective drug costs; thus, it appears that antibiotic resistance associated with a higher financial burden. The result is consistent with the study of Bartsch et al. [22]. In our study, although the mortality of the CREC group was significantly higher than that of the CSEC and no infection groups, mortality was not associated with carbapenem resistance [23].

In our study, the univariate analyses of the two case-control groups found many common risk factors, including prior hospital stay, invasive procedures such as urinary catheter insertion [24], incision of trachea, central venous catheter insertion, and gastric tube insertion, urinary system disease, and antibiotic exposure (cephalosporins, carbapenems, antifungal agents, glycopeptides and oxazolidinones). In addition, our study identified unique risk factors, for example, related laboratory results including low hemoglobin, low blood albumin, and high blood glucose. Multivariate analysis demonstrated a number of risk factors, including prior hospital stay ( $<6$ months), tracheostomy, 
Table 2 Univariate and multivariate analyses regarding the risk factors of the carbapenem-resistant E.coli (CREC) and carbapenemsusceptible E.coli (CSEC) groups

\begin{tabular}{|c|c|c|c|c|c|c|c|c|}
\hline \multirow[t]{2}{*}{ Variable } & \multicolumn{3}{|l|}{ Study group } & \multicolumn{2}{|l|}{ Univariate } & \multicolumn{3}{|c|}{ Multivariable } \\
\hline & Case $(n=49)$ & Control $(n=98)$ & OR & $95 \% \mathrm{Cl}$ & $P$ & $\overline{\mathrm{OR}}$ & $95 \% \mathrm{Cl}$ & $P$ \\
\hline \multicolumn{9}{|l|}{ Demographic characteristics } \\
\hline Sex, male (\%) & $20(41 \%)$ & $40(41 \%)$ & & & 0.57 & & & \\
\hline Age $\{$ year, median (range)\} & $51(0-82)$ & $53(0-91)$ & & & 0.69 & & & \\
\hline \multicolumn{9}{|l|}{ Related to hospitalization } \\
\hline Prior hospital stay ( $<6$ months) & $8(77 \%)$ & $57(58 \%)$ & 2.48 & $1.10-5.56$ & 0.03 & 3.96 & $1.26-12.42$ & 0.02 \\
\hline ICU stay (<6 months) & $18(36 \%)$ & $22(22 \%)$ & 1.48 & $0.98-2.24$ & 0.06 & & & \\
\hline Operation history & $26(53 \%)$ & $28(29 \%)$ & 2.53 & $1.28-5.01$ & 0.01 & & & \\
\hline Urinary catheter insertion & $32(65 \%)$ & $59(60 \%)$ & 1.55 & $1.04-2.32$ & 0.03 & & & \\
\hline Mechanical ventilation & $16(32 \%)$ & $18(18 \%)$ & 1.80 & $0.97-3.35$ & 0.06 & & & \\
\hline Tracheostomy & $12(24 \%)$ & $10(10 \%)$ & 1.64 & $1.09-2.45$ & 0.02 & 2.24 & $1.14-4.38$ & 0.02 \\
\hline Bronchofibroscope use & $7(14 \%)$ & $0(0 \%)$ & 72.96 & $0.53-9980.04$ & 0.09 & & & \\
\hline Central venous catheter insertion & $15(30 \%)$ & $7(7 \%)$ & 4.48 & $1.72-11.67$ & 0.00 & 8.15 & $2.31-28.72$ & 0.00 \\
\hline Gastric tube insertion & $28(57 \%)$ & 37 (37\%) & 1.53 & $1.09-2.16$ & 0.01 & & & \\
\hline Wound drainage tube use & $18(36 \%)$ & $27(27 \%)$ & 1.46 & $0.93-2.29$ & 0.09 & & & \\
\hline \multicolumn{9}{|l|}{ Underlying disorder } \\
\hline Central nervous diseases & 17 (34\%) & $35(35 \%)$ & 0.96 & $0.47-1.96$ & 0.52 & & & \\
\hline Respiratory diseases & $7(14 \%)$ & $18(18 \%)$ & 0.74 & $0.28-1.92$ & 0.35 & & & \\
\hline Circulatory diseases & $11(22 \%)$ & $24(24 \%)$ & 0.89 & $0.39-2.01$ & 0.48 & & & \\
\hline Endocrine diseases & $7(14 \%)$ & $11(11 \%)$ & 1.32 & $0.48-3.64$ & 0.39 & & & \\
\hline Hematological diseases & $3(6 \%)$ & $7(7 \%)$ & 0.85 & $0.21-3.43$ & 0.56 & & & \\
\hline Digestive system diseases & $9(18 \%)$ & $23(23 \%)$ & 0.73 & $0.31-1.74$ & 0.31 & & & \\
\hline Urinary system diseases & $11(36 \%)$ & $8(27 \%)$ & 3.61 & $1.23-10.61$ & 0.02 & 16.69 & $3.01-89.76$ & 0.00 \\
\hline Autoimmune diseases & $3(6 \%)$ & $5(5 \%)$ & 1.21 & $0.28-5.30$ & 0.54 & & & \\
\hline Burn & $10(20 \%)$ & $10(10 \%)$ & 2.26 & $0.87-5.86$ & 0.08 & & & \\
\hline \multicolumn{9}{|l|}{ Antimicrobials agents exposure } \\
\hline Cephalosporins $^{a}$ & $36(73 \%)$ & $52(53 \%)$ & 2.45 & $1.16-5.18$ & 0.01 & & & \\
\hline Carbapenems $^{\mathrm{b}}$ & $19(38 \%)$ & $19(19 \%)$ & 1.91 & $1.19-3.04$ & 0.01 & & & \\
\hline Antifungal agents ${ }^{c}$ & $17(35 \%)$ & $9(9 \%)$ & 1.63 & $1.15-2.32$ & 0.01 & & & \\
\hline Anti-anaerobic agents $^{d}$ & $2(4 \%)$ & $3(3 \%)$ & 1.34 & $0.22-8.34$ & 0.54 & & & \\
\hline Glycopeptides ${ }^{\mathrm{e}}$ and Oxazolidinones & $13(26 \%)$ & $10(10 \%)$ & 1.73 & $1.08-2.78$ & 0.02 & & & \\
\hline \multicolumn{9}{|l|}{ Relative laboratory results } \\
\hline Hemoglobin & $104 \pm 26$ & $114 \pm 26$ & 1.71 & $1.13-2.59$ & 0.01 & 2.83 & $1.46-5.50$ & 0.00 \\
\hline Serum creatinine & $116 \pm 151$ & $115 \pm 25$ & 2.85 & $0.85-9.60$ & 0.09 & & & \\
\hline Blood albumin & $32 \pm 7$ & $36 \pm 7$ & 1.65 & $1.05-2.57$ & 0.03 & & & \\
\hline Blood glucose & $9 \pm 7$ & $6 \pm 3$ & 2.59 & $1.16-5.77$ & 0.02 & 7.01 & $1.89-26.02$ & 0.00 \\
\hline
\end{tabular}

NOTE. Categorical variables are no/total no (\%), and continuous variables are mean \pm SD.Cl:confidence interval, OR:odds ratio

${ }^{a}$ Cephalosporins include First, second, third and fourth generation cephalosporins

${ }^{\mathrm{b}}$ Carbapenems include imipenem, meropenem, and ertapenem

cAntifungal agents include metronidazole and tinidazole

${ }^{d}$ Anti-anaerobic agents include fluconazole, itraconazole, voriconazole and caspofungin

e Glycopeptides include vancomycin, teicoplanin, and norvancomycin

urinary catheter insertion, central venous catheter insertion, carbapenem exposure, urinary system disease, low hemoglobin, and high blood glucose.
The identification of prior hospital stay as risk factor is not unexpected [25]. The environment plays an important role in the spread of antimicrobial resistance, which 
Table 3 Univariate and multivariate analyses regarding the risk factors of the carbapenem-resistant E.coli (CREC) and no infection groups

\begin{tabular}{|c|c|c|c|c|c|c|c|c|}
\hline \multirow[t]{2}{*}{ Variable } & \multicolumn{3}{|l|}{$\underline{\text { Study group }}$} & \multicolumn{2}{|l|}{ Univariate } & \multicolumn{3}{|c|}{$\underline{\text { Multivariable }}$} \\
\hline & Case $(n=49)$ & Control $(n=98)$ & OR & $95 \% \mathrm{Cl}$ & $P$ & $\mathrm{OR}$ & $95 \% \mathrm{Cl}$ & $P$ \\
\hline \multicolumn{9}{|l|}{ Demographic characteristics } \\
\hline Sex, male (\%) & $20(41 \%)$ & $37(38 \%)$ & & & 0.43 & & & \\
\hline Age $\{$ year, median (range)\} & $51(0-82)$ & $47(0-82)$ & & & 0.34 & & & \\
\hline \multicolumn{9}{|l|}{ Related to hospitalization } \\
\hline Prior hospital stay (<6 months) & $38(78 \%)$ & $59(60 \%)$ & 2.36 & $1.05-5.30$ & 0.04 & & & \\
\hline ICU stay (<6 months) & $18(37 \%)$ & $10(10 \%)$ & 3.05 & $1.60-5.82$ & 0.00 & & & \\
\hline Operation history & $26(53 \%)$ & $28(29 \%)$ & 2.53 & $1.28-5.01$ & 0.01 & & & \\
\hline Urinary catheter insertion & $32(65 \%)$ & $19(19 \%)$ & 5.34 & $2.58-11.06$ & 0.00 & 7.14 & $2.37-21.49$ & 0.00 \\
\hline Mechanical ventilation & $16(33 \%)$ & $18(18 \%)$ & 12.45 & $2.92-53.05$ & 0.00 & & & \\
\hline Tracheostomy & $12(24 \%)$ & $1(1 \%)$ & 7.45 & $1.33-41.65$ & 0.02 & & & \\
\hline Central venous catheter insertion & $15(31 \%)$ & $1(1 \%)$ & 7.17 & $2.01-25.60$ & 0.00 & 8.85 & $1.04-75.51$ & 0.04 \\
\hline Bronchofibroscope use & $7(14 \%)$ & $2(2 \%)$ & 6.74 & $1.52-29.83$ & 0.01 & & & \\
\hline Gastric tube insertion & $28(57 \%)$ & $4(4 \%)$ & 19.25 & $2.77-133.69$ & 0.00 & & & \\
\hline Wound drainage tube use & $18(37 \%)$ & $27(28 \%)$ & 3.04 & $1.60-5.78$ & 0.00 & & & \\
\hline \multicolumn{9}{|l|}{ Underlying disorder } \\
\hline Central nervous diseases & 17 (34\%) & $23(23 \%)$ & 1.73 & $0.82-3.67$ & 0.11 & & & \\
\hline Respiratory diseases & $7(14 \%)$ & $13(13 \%)$ & 1.09 & $0.41-2.63$ & 0.53 & & & \\
\hline Circulatory diseases & $11(22 \%)$ & $21(21 \%)$ & 1.06 & $0.46-2.43$ & 0.52 & & & \\
\hline Endocrine diseases & $7(14 \%)$ & $8(8 \%)$ & 1.88 & $0.64-5.51$ & 0.19 & & & \\
\hline Hematological diseases & $3(6 \%)$ & $10(10 \%)$ & 0.57 & $0.15-2.19$ & 0.31 & & & \\
\hline Digestive system diseases & $9(18 \%)$ & $16(16 \%)$ & 1.15 & $0.47-2.84$ & 0.46 & & & \\
\hline Urinary system diseases & $9(18 \%)$ & $8(28 \%)$ & 5.06 & $1.37-18.76$ & 0.02 & 16.79 & $0.72-389.5$ & 0.07 \\
\hline Autoimmune diseases & $3(6 \%)$ & $4(4 \%)$ & 1.53 & $0.33-7.13$ & 0.01 & & & \\
\hline Burn & $10(20 \%)$ & 18.54 & 18.54 & $2.36-145.58$ & 0.01 & & & \\
\hline \multicolumn{9}{|l|}{ Antimicrobials agents exposure } \\
\hline Cephalosporins $^{\text {a }}$ & $36(73 \%)$ & $28(29 \%)$ & 6.92 & $3.20-14.97$ & 0.00 & & & \\
\hline Carbapenems $^{\mathrm{b}}$ & 19 (38\%) & $4(4 \%)$ & 7.41 & $2.46-22.36$ & 0.00 & 12.02 & $1.52-95.4$ & 0.01 \\
\hline Antifungal agents ${ }^{c}$ & 17 (35\%) & $2(2 \%)$ & 4.72 & $1.65-13.52$ & 0.00 & & & \\
\hline Anti-anaerobic agents $^{d}$ & $2(4 \%)$ & $3(3 \%)$ & 1.35 & $0.22-8.34$ & 0.54 & & & \\
\hline Glycopeptides ${ }^{\mathrm{e}}$ and Oxazolidinones & $13(27 \%)$ & $0(0 \%)$ & 4.69 & $1.53-4.31$ & 0.01 & & & \\
\hline \multicolumn{9}{|l|}{ Relative laboratory results } \\
\hline White blood cellcount & $11 \pm 7$ & $7 \pm 4$ & 1.95 & $1.11-3.43$ & 0.00 & & & \\
\hline Hemoglobin & $104 \pm 26$ & $122 \pm 23$ & 2.25 & $1.41-3.57$ & 0.00 & & & \\
\hline Blood albumin & $32 \pm 7$ & $40 \pm 5$ & 4.03 & $2.15-7.58$ & 0.00 & & & \\
\hline Blood glucose & $9 \pm 7$ & $5.5 \pm 1.8$ & 5.29 & $2.09-13.41$ & 0.00 & & & \\
\hline
\end{tabular}

NOTE.Categorical variables are no/total no (\%), and continuous variables are mean \pm SD.Cl:confidence interval, OR:odds ratio

${ }^{a}$ Cephalosporins include First, second, third and fourth generation cephalosporins

${ }^{\mathrm{b}}$ Carbapenems include imipenem, meropenem, and ertapenem

'Antifungal agents include metronidazole and tinidazole

${ }^{\mathrm{d} A n t i-a n a e r o b i c ~ a g e n t s ~ i n c l u d e ~ f l u c o n a z o l e, ~ i t r a c o n a z o l e, ~ v o r i c o n a z o l e ~ a n d ~ c a s p o f u n g i n ~}$

e Glycopeptides include vancomycin, teicoplanin, and norvancomycin

is a limitless reservoir of antimicrobial resistance genes [26]. Patients who fulfill the variables of prior hospital stay and long total hospitalization time may have had more opportunities to be exposed to additional antibiotics and to other patients carrying antibiotic-resistant organisms [27]. Our result is in agreement with those 
Table 4 Economic burden and mortality rate of the three groups

\begin{tabular}{|c|c|c|c|c|c|}
\hline & Case (¥) & control $1(¥)$ & control 2 (¥) & $p 1$ & $p 2$ \\
\hline Mortality & $6 / 49(12 \%)$ & 1/96 (1\%) & $1 / 96(1 \%)$ & 0.01 & 0.01 \\
\hline Total costs & 78,900 & 64,078 & 17,551 & 0.05 & 0.00 \\
\hline examination costs & 2923 & 2571 & 1062 & 0.59 & 0.00 \\
\hline Medical test costs & 6329 & 4649 & 1389 & 0.03 & 0.00 \\
\hline Total drug costs & 42,586 & 29,051 & 6560.5 & 0.03 & 0.00 \\
\hline Anti-infective drug costs & 8907 & 4820 & 122 & 0.01 & 0.00 \\
\hline
\end{tabular}

NOTE. Categorical variables are no/total no (\%), continuous variables are median, case is carbapenem-resistant E.coli (CREC) , control 1 indicates the carbapenemsusceptible E.coli (CSEC) group, and $p 1$ indicates the $\mathrm{p}$ values for the comparison between carbapenem-resistant E.coli (CREC) and carbapenem-susceptible E.coli (CSEC). Control 2 indicates the no infection group, and $p 2$ indicates the $p$ values for the comparison between carbapenem-resistant $E$.coli (CREC) group and no infection group

of a previous study on antibiotic-resistant organisms, which also found these variables to be risk factors [28]. The results suggest that we need to strengthen the management of antibiotics for long-term inpatients and frequently hospitalized patients.

From these two comparisons, it is not surprising to find that invasive procedures, including urinary catheter insertion [24], incision of trachea, and central venous catheter insertion [29] are risk factors for the acquisition of CREC infection. This emphasizes the importance of safety practice in patient care, especially the management of devices. For example, the aseptic technique in catheter use is important as a strategy for the prevention of CREC infections.

There is a close association between healthcareassociated infection and antibiotic use [30-33], especially carbapenem exposure. Thus, in order to more accurately characterize the antibiotic exposure in our study, we assessed the treatment with antibiotics in the 3 months before infection for the case patients and control patients, in this timeframe for data collection is longer than that of other studies [4]. Our findings are in line with those of a recent study that showed the benefit of short-duration, high-dose antibiotic courses as a method to limit unnecessary antibiotic exposure, thus, reduce the risk of antibiotic resistance [34]. According to the suggestion, treatment with high doses and controlled durations is recommended to limit the risk of infections.

It is interesting that the related laboratory results including low hemoglobin and high blood glucose are risk factors for CREC infection, which is different from other studies. The low hemoglobin and high blood glucose are susceptibility risk factors for infection; therefore, special attention should be paid to patients that meet these criteria. We can closely monitor the infection index of these patients while reducing the exposure to risk factors for infection.

One limitation of our study is that we could not assess the patient-to-patient infection spread, we did not collect isolates for gene molecular epidemiologic analysis, thus, we could not assess if there were any outbreaks during the study period. The second is the small study sample size. Moreover, the financial burden is associated with total cost of patients after isolation of CREC or CSEC, of which the direct cost of CREC or CSEC infection was not considered.

\section{Conclusion}

Our results suggest that healthcare-acquired CREC infection may be related to prior hospital stay, tracheostomy, central venous catheter insertion, carbapenem exposure, and urinary system disease. Further, anemia and high blood glucose are important risk factors for the acquisition of CREC infection. Hospital infection control and the implementation of antimicrobial stewardship practices across the continuum of healthcare settings will hopefully help to curb the emergence and spread of CREC infections.

\section{Abbreviations}

CAl: Community-associated infection.; CRE: Carbapenem-resistant Enterobacteriaceae; CREC: Carbapenem-resistant Escherichia coli; CSEC: Carbapenem-susceptible escherichia coli; HAl: Healthcare-associated infection; ICU: Intensive care unit

\section{Acknowledgements}

The authors thank all members of Infection Control Center of Xiangya Hospital for the technical supports and language proof reading.

\section{Funding}

This work was supported by the Research Fund of Hunan Provincial Health and Family Planning Commission (No. B2016107), Young Scientists Fund of Xiangya Hospital (2014Q05), and Xiangya Sinobio way Health Research Fund (No. xywm2015111).

\section{Availability of data and materials}

The datasets supporting the conclusions of this article are included within the article.

\section{Author' contributions}

XJM, CHL and AHW conceived the experiments, XJM, SDL, JPD, XH, PCZ, XRX REG, YZ, and CCF conducted the experiments, and XJM analyzed the results. XJM wrote the draft manuscript. XJM, CHL and AHW finalized the manuscript. All authors reviewed and approved the final manuscript.

\section{Authors' information}

All of the author come from infection Control Center of Xiangya Hospital Central South University in China. 


\section{Competing interests}

The authors declare that they have no competing interests.

\section{Consent for publication}

Not applicable.

\section{Ethics approval and consent to participate}

This study was approved by the Ethics Committee of Xiangya Hospital Central South University (NO 201,510,052) and all participates consent to join in the study.

\section{Received: 16 September 2016 Accepted: 27 December 2016} Published online: 17 January 2017

\section{References}

1. Feng Y, Yang P, Xie Y, Wang X, McNally A, Zong Z. Escherichia coli ofsequence type 3835 carrying bla NDM-1, bla CTX-M-15, bla CMY-42 and bla SHV-12. Sci Rep. 2015;5:12275.

2. Lartigue MF, Poirel L, Poyart C, Reglier-Poupet H, Nordmann P. Ertapenem resistance of Escherichia coli. Emerg Infect Dis. 2007;13(2):315-7.

3. Lee BY, Bartsch SM, Wong KF, McKinnell JA, Slayton RB, Miller LG, Cao C, Kim DS, Kallen AJ, Jernigan JA, et al. The Potential Trajectory of Carbapenem-Resistant Enterobacteriaceae, an Emerging Threat to HealthCare Facilities, and the Impact of the Centers for Disease Control and Prevention Toolkit. Am J Epidemiol. 2016;183(5):471-9.

4. Jeon M, Choi S, Kwak YG, Chung J, Lee S, Jeong J, Woo JH, Kim YS. Risk factors for the acquisition of carbapenem-resistant Escherichia coli among hospitalized patients. Diagn Microbiol Infect Dis. 2008;62(4):402-6.

5. Gulmez D, Woodford N, Palepou MF, Mushtaq S, Metan G, Yakupogullari Y, Kocagoz S, Uzun O, Hascelik G, Livermore DM. Carbapenem-resistant Escherichia coli and Klebsiella pneumoniae isolates from Turkey with OXA48-like carbapenemases and outer membrane protein loss. Int J Antimicrob Agents. 2008;31(6):523-6.

6. Urban C, Bradford PA, Tuckman M, Segal Maurer S, Wehbeh W, Grenner L, Colon Urban R, Mariano N, Rahal JJ. Carbapenem-ResistantEscherichia coli HarboringKlebsiella pneumoniae Carbapenemase $\beta$-Lactamases Associated with Long-Term Care Facilities. Clin Infect Dis. 2008;46(11):e127-30.

7. Cui L, Zhao J, Lu J. Molecular characteristics of extended spectrum betalactamase and carbapenemase genes carried by carbapenem-resistant Enterobacter cloacae in a Chinese university hospital. Turkish Gunma J Med Sci. 2015;45(6):1321-8.

8. Queenan AM, Bush K. Carbapenemases: the versatile beta-lactamases. Clin Microbiol Rev. 2007;20(3):440-58.

9. Cantón R, Akóva M, Carmeli Y, Giske CG, Glupczynski Y, Gniadkowski M, Livermore DM, Miriagou V, Naas T, Rossolini GM, et al. Rapid evolution and spread of carbapenemases among Enterobacteriaceae in Europe. Clin Microbiol Infect. 2012;18(5):413-31.

10. Shevchenko OV, Mudrak DY, Skleenova EY, Kozyreva VK, llina EN, Ikryannikova LN, Alexandrova IA, Sidorenko SV, Edelstein MV. First detection of VIM-4 metallo-beta-lactamase - producing Escherichia coli in Russia. Clin Microbiol Infect. 2012;18(7):E214-7.

11. Nordmann P, Naas T, Poirel L. Global spread of Carbapenemase-producing Enterobacteriaceae. Emerg Infect Dis. 2011;17(10):1791-8.

12. Mushtaq S, Irfan S, Sarma JB, Doumith M, Pike R, Pitout J, Livermore DM, Woodford N. Phylogenetic diversity of Escherichia coli strains producing NDM-type carbapenemases. J Antimicrob Chemother. 2011;66(9):2002-5.

13. Glasner C, Albiger B, Buist G, Tambic AA, Canton R, Carmeli Y, Friedrich AW, Giske CG, Glupczynski Y, Gniadkowski M, et al. Carbapenemase-producing Enterobacteriaceae in Europe: a survey among national experts from 39 countries, February 2013. Euro Surveill. 2013;18(28):9-15.

14. Tzouvelekis LS, Markogiannakis A, Psichogiou M, Tassios PT, Daikos GL. Carbapenemases in Klebsiella pneumoniae and other Enterobacteriaceae: an evolving crisis of global dimensions. Clin Microbiol Rev. 2012;25(4):682-707.

15. Schwaber MJ, Carmeli Y. Carbapenem-resistant Enterobacteriaceae: a potential threat. JAMA. 2008;300(24):2911-3.

16. Teo J, Cai Y, Tang S, Lee W, Tan TY, Tan TT, Kwa AL. Risk factors, molecular epidemiology and outcomes of ertapenem-resistant, carbapenemsusceptible Enterobacteriaceae: a case-case-control study. Plos One. 2012; 7(3):e34254.

17. Nguyen ML, Toye B, Kanji S, Zvonar R. Risk Factors for and Outcomes of Bacteremia Caused by Extended-Spectrum ss-Lactamase-Producing
Escherichia coli and Klebsiella Species at a Canadian Tertiary Care Hospital. Can J Hosp Pharm. 2015;68(2):136-43.

18. Paterson DL. Looking for risk factors for the acquisition of antibiotic resistance: a 21st-century approach. Clin Infect Dis. 2002;34(12):1564-7.

19. Kaye KS, Harris AD, Samore M, Carmeli Y. The case-case-control study design: addressing the limitations of risk factor studies for antimicrobial resistance. Infect Control Hosp Epidemiol. 2005;26(4):346-51.

20. Logan LK, Meltzer LA, McAuley JB, Hayden MK, Beck T, Braykov NP, Laxminarayan R, Weinstein RA. Extended-Spectrum beta-LactamaseProducing Enterobacteriaceae Infections in Children: A Two-Center CaseCase-control Study of Risk Factors and Outcomes in Chicago. Illinois. J Pediatric Infect Dis Soc. 2014;3(4):312-9.

21. Suwantarat N, Carroll KC. Epidemiology and molecular characterization of multidrug-resistant Gram-negative bacteria in Southeast Asia. Antimicrob Resist Infect Control. 2016;5(1):1-8.

22. Bartsch SM, McKinnell JA, Mueller LE, Miller LG, Gohil SK, Huang SS, Lee BY: Potential economic burden of carbapenem-resistant Enterobacteriaceae (CRE) in the United States. Clin Microbiol Infect. 2016;23(1):48.e9-48.e16.

23. Candevir UA, Kurtaran B, Inal AS, Komur S, Kibar F, Yapici CH, Bozkurt S, Gurel D, Kilic F, Yaman A, et al. Risk factors of carbapenem-resistant Klebsiella pneumoniae infection: a serious threat in ICUs. Med Sci Monit. 2015;21:219-24.

24. Ling ML, Tee YM, Tan SG, Amin IM, How KB, Tan KY, Lee LC. Risk factors for acquisition of carbapenem resistant Enterobacteriaceae in an acute tertiary care hospital in Singapore. Antimicrob Resist Infect Control. 2015;4(1):1-7.

25. Han JH, Nachamkin I, Zaoutis TE, Coffin SE, Linkin DR, Fishman NO, Weiner MG, Hu B, Tolomeo P, Lautenbach E. Risk factors for gastrointestinal tract colonization with extended-spectrum beta-lactamase (ESBL)-producing Escherichia coli and Klebsiella species in hospitalized patients. Infect Control Hosp Epidemiol. 2012;33(12):1242-5.

26. Gonzalez-Zorn B, Escudero JA. Ecology of antimicrobial resistance: humans, animals, food and environment. INT MICROBIOL. 2012;15(3):101-9.

27. Zhou P, Xiong X, Li C, Wu A. Association of Length of Stay With Contamination of Multidrug-Resistant Organisms in the Environment and Colonization in the Rectum of Intensive Care Unit Patients in China. Infect Control Hosp Epidemiol. 2016;37(1):120-1.

28. Harris AD, Smith D, Johnson JA, Bradham DD, Roghmann MC. Risk Factors for Imipenem-Resistant Pseudomonas aeruginosa among Hospitalized Patients. CLIN INFECT DIS. 2002;34(3):340-5.

29. Kang Cl, Wi YM, Lee MY, Ko KS, Chung DR, Peck KR, Lee NY, Song JH. Epidemiology and risk factors of community onset infections caused by extended-spectrum beta-lactamase-producing Escherichia coli strains. J CLIN MICROBIOL. 2012;50(2):312-7.

30. Cheah AL, Peel T, Howden BP, Spelman D, Grayson ML, Nation RL, Kong DC Case-case-control study on factors associated with vanB vancomycin-resistant and vancomycin-susceptible enterococcal bacteraemia. BMC Infect Dis. 2014; $14(1): 1-8$.

31. Kritsotakis El, Tsioutis C, Roumbelaki M, Christidou A, Gikas A. Antibiotic use and the risk of carbapenem-resistant extended-spectrum-\{beta\}-lactamaseproducing Klebsiella pneumoniae infection in hospitalized patients: results of a double case-control study. J Antimicrob Chemother. 2011;66(6):1383-91.

32. Patel N, Harrington S, Dihmess A, Woo B, Masoud R, Martis P, Fiorenza M, Graffunder E, Evans A, McNutt LA, et al. Clinical epidemiology of carbapenem-intermediate or -resistant Enterobacteriaceae. J Antimicrob Chemother. 2011;66(7):1600-8.

33. Liu SW, Chang HJ, Chia JH, Kuo AJ, Wu TL, Lee MH. Outcomes and characteristics of ertapenem-nonsusceptible Klebsiella pneumoniae bacteremia at a university hospital in Northern Taiwan: a matched case-control study. J Microbiol Immunol Infect. 2012;45(2):113-9.

34. Stein RA. When less is more: high-dose, short duration regimens and antibiotic resistance. INT J CLIN PRACT. 2008;62(9):1304-5. 